\title{
Inhaltsverzeichnis
}

Editorial: Grenzenlose Werbung zwischen Konsum und Audiovision — VII

Dirk Schindelbeck

Julius Pinschewer und der gezeichnete Werbetrickfilm in Deutschland

1897-1933 - 1

Anne Schmidt

Angsterzeugung oder Glücksversprechen. Über reklametaugliche Gefühle im

Kino -30

\section{Sebastian Thalheim}

Sommer, Sonne, Schmalfilm. Die neue Visualisierung eines alten Mediums.

Werbefilm im Kontext der Konsumkultur der DDR in den 1950er Jahren — 53

Solveig Ottmann / Sandra Reimann

Audiovision in drei Teilen. Analysen zur Werbeschallplatte unter besonderer Berücksichtigung des Beispiels Was ist Hi-Fi? der Marke

Bang \& Olufsen (1965) - 79

Karin Moser

Begrenzte Grenzenlosigkeit: Inhaltliche Konzeption und filmische Strategien der Werbefilme der Austria Tabak 1948-2000 -109

\section{Bernhard Dietz}

Revolte in der Warenwelt. Konsumkritik, nonkonformistische Ästhetik und der Paradigmenwechsel in der westdeutschen Werbeindustrie von 1968-86 145

Mario Keller

„Provokant und in keiner Weise tragbar“: Die Humanic-Werbung der 1970er Jahre als Grenzerfahrung zwischen Avantgardekunst und Werbung - 172

Gabriele Fröschl

Das Audiovisuelle im Archiv — 203

Stephanie Scholz

Das Geschäft mit den Spielzeughelden. Kinderfernsehen zwischen Pädagogik und Program-Length Commercials — 227 
Michael Cowan

Public Advertising Screens and the Ambivalence of Interactivity 260

Guido Zurstiege

Digitale Transformation der Werbung - Herausforderung für die

Werbeforschung - 286

Autor*innenverzeichnis - 313 